\title{
Measuring the Irregularity of Vector-Valued Morphological Operators using Wasserstein Metric ${ }^{\star}$
}

\author{
Marcos Eduardo Valle ${ }^{1}$, Samuel Francisco ${ }^{1,2}$, \\ Marco Aurélio Granero ${ }^{2}$, and Santiago Velasco-Forero ${ }^{3}$ \\ ${ }^{1}$ Universidade Estadual de Campinas, Campinas - SP, Brazil. \\ ${ }^{2}$ Instituto Federal de Educação, Ciência e Tecnologia de São Paulo, São Paulo - SP, Brazil. \\ ${ }^{3}$ Center of Mathematical Morphology, Mines ParisTech, PSL Research University, France
}

\begin{abstract}
Mathematical morphology is a useful theory of nonlinear operators widely used for image processing and analysis. Despite the successful application of morphological operators for binary and gray-scale images, extending them to vector-valued images is not straightforward because there are no unambiguous orderings for vectors. Among the many approaches to multivalued mathematical morphology, those based on total orders are particularly promising. Morphological operators based on total orders do not produce the so-called false-colors. On the downside, they often introduce irregularities in the output image. Although the irregularity issue has a rigorous mathematical formulation, we are not aware of an efficient method to quantify it. In this paper, we propose to quantify the irregularity of a vector-valued morphological operator using the Wasserstein metric. The Wasserstein metric yields the minimal transport cost for transforming the input into the output image. We illustrate by examples how to quantify the irregularity of vector-valued morphological operators using the Wasserstein metric.
\end{abstract}

Keywords: mathematical morphology $\cdot$ vector-valued images $\cdot$ total order $\cdot$ irregularity issue $\cdot$ optimal transportation.

\section{Introduction}

Mathematical morphology (MM) is a nonlinear theory that uses geometric and topological concepts for image and signal processing. The theory of mathematical morphology is usually defined on an algebraic structure called complete lattices which is satisfactory for binary and grayscale images $[10,8]$. In the case of vector-valued images, vector spaces endowed with a total order is one of the most comfortable frameworks for the extension of morphological processing [2,1,22]. Approaches that have been recently formulated using total orderings include [9,12,18-21]. Despite their successful applications for color and hyperspectral image processing, Chevallier and Angulo showed that the information contained in a total order is too weak to reproduce the natural topology of the value space [5]. As a consequence, morphological operators may introduce irregularities and aliasing on images.

\footnotetext{
* This work was supported in part by the São Paulo Research Foundation (FAPESP) under grant no 2019/02278-2 and the National Council for Scientific and Technological Development (CNPq) under grant no 310118/2017-4.
} 
Our motivation is to formulate quantitative measures to study the irregularity implied by a morphological operator on vector-valued images. We believe that this is the first work proposing a framework based on the Wasserstein metric to score this irregularity effect considering pairs of input/output images $[11,23]$. The paper is organized as follows: Section 2 introduced mathematical morphology concepts for vector-valued images and the difficulties produced by operators based on total orders. Section 3 presents our proposition of irregularity measure. Additionally, results on natural images show the goodness of proposed measures. The paper ends with Section 4 including conclusions and recommendations for future works.

\section{Basic Concepts on Mathematical Morphology}

Let us begin by presenting the basic concepts and the notations used in this paper. First, an image $\mathbf{I}$ corresponds to a mapping from a point set $D$ to a value set $\mathbb{V}$, that is, I : $D \rightarrow \mathbb{V}$. The set of all images from a domain $D$ to $\mathbb{V}$ is denoted by $\mathcal{V}=\mathbb{V}^{D}$. Throughout the paper, we assume the point set $D$ is finite and included in a space $\mathcal{E}$, where $(\mathcal{E},+)$ is a group. Usually, we consider $\mathcal{E}=\mathbb{R}^{2}$ or $\mathcal{E}=\mathbb{Z}^{2}$ with the usual addition. Furthermore, we assume the value set $\mathbb{V}$ is a complete lattice equipped with a metric $d: \mathbb{V} \times \mathbb{V} \rightarrow[0,+\infty)$. Recall that a complete lattice $\mathbb{L}$ is a partially ordered set in which any subset $X \subset \mathbb{L}$ has both an infimum and a supremum [3]. The infimum and the supremum of $X$ are denoted respectively by $\wedge X$ and $\bigvee X$.

\subsection{Mathematical Morphology on Complete Lattices}

Mathematical morphology (MM) is mainly concerned with image operators used to extract relevant geometric and topological information from an image $[8,6,15]$. The two elementary operators of MM are dilations and erosions. Many other operators, such as opening, closing, and the morphological gradient, are obtained by combining the elementary morphological operators.

Complete lattices provide an appropriate mathematical background for defining the elementary morphological operators $[10,8]$. Indeed, the elementary morphological operators are those that commute with the supremum and the infimum operations in a complete lattice. When the value set $\mathbb{V}$ is a complete lattice, the operators $\delta_{S}, \varepsilon_{S}: \mathcal{V} \rightarrow \mathcal{V}$ given by the following equations where $S \subseteq \mathcal{E}$ is finite are respectively a dilation and an erosion:

$$
\delta_{S}(\mathbf{I})(p)=\bigvee_{\substack{s \in S \\ p-s \in D}} \mathbf{I}(p-s) \quad \text { and } \quad \varepsilon_{S}(\mathbf{I})(p)=\bigwedge_{\substack{s \in S \\ p+s \in D}} \mathbf{I}(p+s) .
$$

The set $S$ is referred to as the structuring element (SE) [15]. The images $\delta_{S}(\mathbf{I})$ and $\varepsilon_{S}(\mathbf{I})$ are respectively the dilation and the erosion of $\mathbf{I}$ by the structuring element $S$.

Although there exist more general definitions, the elementary morphological operators given by (1) are widely used in practical situations. Combining dilations and erosions, we obtain many other morphological operators. In this paper, we focus on elementary operators defined by (1). We also consider openings $\gamma_{S}$ and closings $\phi_{S}$, which are obtained by the compositions $\gamma_{S}=\delta_{S} \circ \varepsilon_{S}$ and $\phi_{S}=\varepsilon_{S} \circ \delta_{S}$ [15]. 


\subsection{Vector-Valued Mathematical Morphology}

Let us now address morphological operators for vector-valued images. A vector-valued image is obtained by considering $\mathbb{V} \subseteq \overline{\mathbb{R}}^{d}$, where $\overline{\mathbb{R}}=\mathbb{R} \cup\{-\infty,+\infty\}$ and $d \geq 2$.

Vector-valued dilations and erosions can be defined using (1) whenever the vectorvalued set $\mathbb{V}$ is a complete lattice. However, there are many different ordering schemes for vector-valued sets. Defining an appropriate ordering scheme is one of the main challenges of vector-valued MM. The following references provide a brief sense of interesting directions of research on vector-valued MM [1, 2, 4, 5, 7, 9, 18, 19, 21, 22].

As examples of ordering schemes on vector-valued sets, let us recall the marginal and the lexicographical orderings [2]. The marginal ordering is defined as follows for all $\boldsymbol{u}=\left(u_{1}, \ldots, u_{d}\right) \in \mathbb{V}$ and $\boldsymbol{v}=\left(v_{1}, \ldots, v_{d}\right) \in \mathbb{V}$ :

$$
\boldsymbol{u} \leq_{M} \boldsymbol{v} \quad \Longleftrightarrow \quad u_{i} \leq v_{i}, \forall i=1, \ldots, d,
$$

where " $\leq$ " denotes the usual ordering on $\mathbb{R}$. The marginal ordering is also called the component-wise ordering or the Cartesian product ordering. The lexicographical ordering is defined as follows:

$$
\boldsymbol{u} \leq_{L} \boldsymbol{v} \quad \Longleftrightarrow \quad \exists i: u_{i} \leq v_{i} \text { and } u_{j}=v_{j}, \forall j<i .
$$

In contrast to the marginal ordering, the lexicographical ordering is a total ordering. Hence, either one of the inequalities $\boldsymbol{u} \leq_{L} \boldsymbol{v}$ or $\boldsymbol{v} \leq_{L} \boldsymbol{u}$ holds for any $\boldsymbol{u}, \boldsymbol{v} \in \mathbb{V}$.

\subsection{The False "Colors" Problem Versus the Irregularity Issue}

One problem on vector-valued MM is the creation of "false colors" or, more generally, false values [14]. A morphological operator $\psi: \mathcal{V} \rightarrow \mathcal{V}$ introduces false values whenever there are values on $\psi(I)$ which do not belong to the original image I. Formally, let $2^{\mathbb{V}}$ denote the power set of $\mathbb{V}$ and let $V: \mathcal{V} \rightarrow 2^{\mathbb{V}}$ be the mapping given by

$$
V(\mathbf{I})=\{\mathbf{I}(p): p \in D\}, \quad \forall \mathbf{I} \in \mathcal{V} .
$$

A morphological operator $\psi$ introduces false colors if the set difference $V(\psi(\mathbf{I})) \backslash V(\mathbf{I})$ is not empty. The abnormal false values can be a problem in many applications such as when dealing with satellite data [14]. Using the marginal ordering, the dilation and the erosion given by (1) usually yield false colors.

A total ordering, such as the lexicographical ordering, circumvents the problem of the false values [14]. Using a total ordering, the supremum and the infimum of a finite set is an element of the set, i.e., they coincide with the maximum and minimum operations, respectively. As a consequence, if $D$ is finite, the elementary morphological operators given by (1) only contain values of the input image $\mathbf{I}$. On the downside, a total ordering can be irregular in a metric space. According to Chevallier and Angulo, the irregularities follow because the topology induced by a total order may not reproduce the topology of a metric space [5]. Specifically, let the value set $\mathbb{V}$ be a totally ordered set as well as a metric space, with metric $d: \mathbb{V} \times \mathbb{V} \rightarrow[0,+\infty)$. Chevallier and Angulo showed that there exists $\boldsymbol{u}, \boldsymbol{v}, \boldsymbol{w} \in \mathbb{V}$ such that $\boldsymbol{u} \leq \boldsymbol{v} \leq \boldsymbol{w}$ but $d(\boldsymbol{u}, \boldsymbol{w})<d(\boldsymbol{u}, \boldsymbol{v})$ 
a) Dilated Image $\mathbf{J}_{L}$ (Lexicographical RGB)

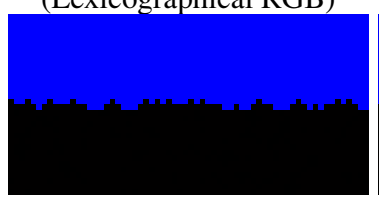

b) Toy Image I

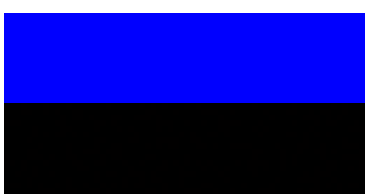

c) Dilated Image $\mathbf{J}_{M}$ (Marginal)

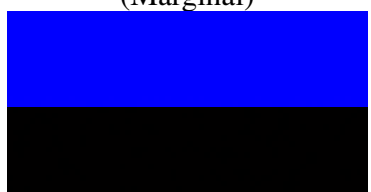

Fig. 1. Illustractive example of the irregularity issue. Image with three colors and its corresponding dilation by a cross structuring element using the RGB lexicographial and marginal orderings.

under mild conditions with respect to the connectivity of $\mathbb{V}$. In words, although $\boldsymbol{w}$ is closer to $\boldsymbol{u}$ than $\boldsymbol{v}$, the inequalities $\boldsymbol{u} \leq \boldsymbol{v} \leq \boldsymbol{w}$ suggest $\boldsymbol{w}$ is farther from $\boldsymbol{u}$ than $\boldsymbol{v}$. Since the morphological operators are defined using the extrema operators, they do not take the metric of $\mathbb{V}$ into account.

A visual interpretation of the irregularity issue is shown in Figure 1, which is very similar to an example provided in [5]. Figure 1b) shows an image with three RGB colors, namely $\boldsymbol{u}=(0,0,0), \boldsymbol{v}=(0,0,1)$, and $\boldsymbol{w}=(0.005,0,0)$. The toy image $\mathbf{I}$ is obtained by replacing pure black values $\boldsymbol{u}$ by $\boldsymbol{w}$ with probability 0.3 from an image of size $32 \times 64$ with two stripes of colors blue and black. The dilations $\mathbf{J}_{L}$ and $\mathbf{J}_{M}$ by a cross as the structuring element using respectively the lexicographical RGB and the marginal ordering schemes are also depicted in Figure 1. Visually, $\boldsymbol{u}$ and $\boldsymbol{w}$ are black colors while $\boldsymbol{v}$ is a pure blue. Using the Euclidean distance, we obtain $d(\boldsymbol{u}, \boldsymbol{v})=$ 1 and $d(\boldsymbol{u}, \boldsymbol{w})=0.005$. These distances agree with our color perception. However, using the lexicographical ordering, we obtain $\boldsymbol{u} \leq_{L} \boldsymbol{v} \leq_{L} \boldsymbol{w}$. As a consequence, the following happens when we compute the dilation $\delta_{S}(\mathbf{I})=\mathbf{J}_{L}$ using the lexicographical ordering: the blue pixel value $\boldsymbol{v}$ advances over the black $\boldsymbol{u}$ but it is overlaid by the black $\boldsymbol{w}$, resulting in the irregularities shown in Figure 1a). In contrast, the dilated image depicted in Figure 1c) obtained using the marginal ordering does not present any visual irregularity.

Although we know the irregularity results from a divergence between the topologies induced by the metric and the total ordering, there is no consensual quantitative measure which agrees with our visual perception. A quantitative measure can help to choose an appropriate ordering scheme for vector-valued mathematical morphology. In the following section, we propose to measure the irregularity using the Wasserstein metric.

\section{Measuring the Irregularity}

In this section, we present a quantitative measure for the irregularity issue, called the irregularity index. We begin by presenting a global irregularity index. Then, we propose computing the irregularity index as an average of local irregularity indexes. Although we are interested in measuring the irregularity implied by a total ordering, we will not assume $\mathbb{V}$ is totally ordered. Indeed, the proposed irregularity measure is well defined whenever $D$ is finite and $\mathbb{V}$ is a metric space. 


\subsection{The Wasserstein Metric and the Generalized Sum of Pixel-Wise Distances}

The global irregularity index is defined in terms of the quotient of the Wasserstein metric and a generalized sum of pixel-wise distances. Let us begin by reviewing the generalized sum of pixel-wise distances.

Consider an image operator $\psi: \mathcal{V} \rightarrow \mathcal{V}$. Given an input image $\mathbf{I} \in \mathcal{V}$, let $\mathbf{J}=\psi(\mathbf{I})$ denote the output of the image operator. The generalized sum of pixel-wise distances of $\mathbf{I}$ and $\mathbf{J}$ is an operator $\mathcal{D}_{p}: \mathcal{V} \times \mathcal{V} \rightarrow[0,+\infty)$ given by

$$
\mathcal{D}_{p}(\mathbf{I}, \mathbf{J})=\left(\sum_{x \in D} d^{p}(\mathbf{I}(x), \mathbf{J}(x))\right)^{\frac{1}{p}}, \quad p \geq 1
$$

The generalized sum of pixel-wise distances is one of the simplest measures that takes into account the metric $d$ and the pixel locations. However, $\mathcal{D}_{p}$ is usually not properly scaled; possibly because its dimension is the same as the metric $d$. For example, using the Euclidean RGB distance and $p=1$, the images shown in Figure 1 yield the values $\mathcal{D}_{1}\left(\mathbf{I}, \mathbf{J}_{L}\right)=34.12$ and $\mathcal{D}_{1}\left(\mathbf{I}, \mathbf{J}_{M}\right)=66.05$. Note that $\mathcal{D}_{1}\left(\mathbf{I}, \mathbf{J}_{L}\right) \leq \mathcal{D}_{1}\left(\mathbf{I}, \mathbf{J}_{M}\right)$. Hence, the generalized sum of pixel-wise distances is not an appropriate quantitative measure for the irregularity issue.

Let us now review the Wasserstein metric, also known as the earth mover's distance or the Kantorovich-Rubinstein distance in certain contexts [11,23]. The Wasserstein metric, named after the Russian mathematician Leonid Vaseršte ̌n, has been previously used by Rubner et al. for content-based image retrieval [11]. In the general case, the Wasserstein metric is used to compute distances between probability distributions. For discrete probabilities, however, it is formulated as a transportation problem.

The objective of a transportation problem is to minimize the cost to deliver items from $n$ factories to $m$ shops. In our context, the transportation problem minimizes the cost to transform the input image $\mathbf{I}$ into the output image $\mathbf{J}$. The cost is defined using the metric on the value set $\mathbb{V}$. Precisely, let $V(\mathbf{I})=\left\{v_{1}, \ldots, v_{n}\right\}$ and $V(\mathbf{J})=$ $\left\{u_{1}, \ldots, u_{m}\right\}$ be the sets of color values of $\mathbf{I}$ and $\mathbf{J}$, respectively. Given $p \geq 1$, the cost to transform a value $v_{i}$ of $\mathbf{I}$ into a value $u_{j}$ of $\mathbf{J}$ is defined by

$$
c_{i j}=d^{p}\left(v_{i}, u_{j}\right), \quad i=1, \ldots, n, j=1, \ldots, m .
$$

The Wasserstein metric, denoted by $\mathcal{W}_{p}: \mathcal{V} \times \mathcal{V} \rightarrow[0, \infty)$ for $p \geq 1$, is given by

$$
\mathcal{W}_{p}(\mathbf{I}, \mathbf{J})=\left(\sum_{i=1}^{n} \sum_{j=1}^{m} c_{i j} x_{i j}\right)^{1 / p}, \quad p \geq 1
$$


where $x_{i j}$ solves the linear programming problem

$$
\left\{\begin{aligned}
\text { minimize } & \sum_{i=1}^{n} \sum_{j=1}^{m} c_{i j} x_{i j} \\
\text { subject to } & \sum_{j=1}^{m} x_{i j}=\operatorname{Card}\left(\left\{x: \mathbf{I}(x)=v_{i}\right\}\right), \quad \forall i=1, \ldots, n, \\
& \sum_{i=1}^{n} x_{i j}=\operatorname{Card}\left(\left\{x: \mathbf{J}(x)=u_{j}\right\}\right), \quad \forall j=1, \ldots, m, \\
& x_{i j} \geq 0, \quad \forall i=1, \ldots, n, \forall j=1, \ldots, m .
\end{aligned}\right.
$$

In the transportation problem (8), the variable $x_{i j}$ represents the amount of the pixel value $v_{i}$ of $\mathbf{I}$ transformed to the pixel value $u_{j}$ of $\mathbf{J}$. In some sense, the Wasserstein metric can be interpreted as the minimal cost to transform $\mathbf{I}$ into $\mathbf{J}$. Considering $p=1$ and the Euclidean distance, we obtain $\mathcal{W}_{1}\left(\mathbf{I}, \mathbf{J}_{L}\right)=6.18$ and $\mathcal{W}_{1}\left(\mathbf{I}, \mathbf{J}_{M}\right)=65.94$ for the images shown in Figure 1. Note that the inequality $\mathcal{W}_{1}\left(\mathbf{I}, \mathbf{J}_{L}\right)<\mathcal{W}_{1}\left(\mathbf{I}, \mathbf{J}_{M}\right)$ holds in this example. Like the generalized sum of pixel-wise distances, the Wasserstein metric is not an appropriate measure of the irregularity; possibly because it has the same dimension as the generalized sum of pixel-wise distances.

\subsection{Global Irregularity Index}

Although both generalized sum of pixel-wise distances and the Wasserstein metric are, per se, not appropriate to evaluate the irregularity issue, we advocate in this paper that their quotient yields a useful measure. First of all, note that the generalized sum of pixel-wise distances satisfies

$$
\mathcal{D}_{p}(\mathbf{I}, \mathbf{J})=\left(\sum_{i=1}^{n} \sum_{j=1}^{m} c_{i j} y_{i j}\right)^{\frac{1}{p}}, \quad p \geq 1,
$$

where

$$
y_{i j}=\operatorname{Card}\left(\left\{x: \mathbf{I}(x)=v_{i} \text { and } \mathbf{J}(x)=u_{j}, x \in D\right\}\right),
$$

for all $i=1, \ldots, n$ and $j=1, \ldots, m$. Moreover, it is not hard to see that $y_{i j} \geq 0$,

$$
\sum_{j=1}^{m} y_{i j}=\operatorname{Card}\left(\left\{x: \mathbf{I}(x)=v_{i}\right\}\right) \quad \text { and } \quad \sum_{i=1}^{n} y_{i j}=\operatorname{Card}\left(\left\{x: \mathbf{J}(x)=u_{j}\right\}\right)
$$

for all $i=1, \ldots, n$ and $j=1, \ldots, m$. Therefore, the generalized sum of pixelwise distances also measures the cost of transforming $\mathbf{I}$ into $\mathbf{J}$. Moreover, $\mathcal{D}_{p}$ and $\mathcal{W}_{p}$ have the same units and magnitudes. Because $\mathcal{W}_{p}$ is the minimal cost, the inequality $\mathcal{W}_{p}(\mathbf{I}, \mathbf{J}) \leq \mathcal{D}_{p}(\mathbf{I}, \mathbf{J})$ holds for any $\mathbf{I}$ and $\mathbf{J}=\psi(\mathbf{I})$. Using these remarks, we propose to measure the irregularity using the mapping $\Phi_{p}^{g}: \mathcal{V} \times \mathcal{V} \rightarrow[0,1]$ given by the relative gap between $\mathcal{D}_{p}$ and $\mathcal{W}_{p}$. Precisely, given images $\mathbf{I}, \mathbf{J} \in \mathcal{V}$, we define the global irregularity index by means of the equation

$$
\Phi_{p}^{g}(\mathbf{I}, \mathbf{J})=\frac{\mathcal{D}_{p}(\mathbf{I}, \mathbf{J})-\mathcal{W}_{p}(\mathbf{I}, \mathbf{J})}{\mathcal{D}_{p}(\mathbf{I}, \mathbf{J})}, \quad \text { if } \quad \mathcal{D}_{p}(\mathbf{I}, \mathbf{J}) \neq 0,
$$


and $\Phi_{p}^{g}(\mathbf{I}, \mathbf{J})=0$ if $\mathcal{D}_{p}(\mathbf{I}, \mathbf{J})=0$. Note that the larger the gap between $\mathcal{W}_{p}(\mathbf{I}, \mathbf{J})$ and $\mathcal{D}_{p}(\mathbf{I}, \mathbf{J})$, the larger the global irregularity index. Equivalently, we have

$$
\Phi_{p}^{g}(\mathbf{I}, \mathbf{J})= \begin{cases}0, & \text { if } \mathcal{D}_{p}(\mathbf{I}, \mathbf{J})=0 \\ 1-\frac{\mathcal{W}_{p}(\mathbf{I}, \mathbf{J})}{\mathcal{D}_{p}(\mathbf{I}, \mathbf{J})}, & \text { otherwise }\end{cases}
$$

The irregularity index is symmetric and bounded, that is, $\Phi_{p}(\mathbf{I}, \mathbf{J})=\Phi_{p}(\mathbf{J}, \mathbf{I})$ and $0 \leq \Phi_{p}(\mathbf{I}, \mathbf{J}) \leq 1$. Moreover, $\Phi_{p}^{g}(\mathbf{I}, \mathbf{J})$ is a dimensionless quantity. The more irregular is $\mathbf{J}=\psi(\mathbf{I})$, the larger the value of $\Phi_{p}^{g}(\mathbf{I}, \mathbf{J})$ is expect to be. For example, using $p=1$ and the Euclidean distance, the irregularity index of the dilated images shown in Figure 1a) and 1c) are respectively $\Phi_{1}^{g}\left(\mathbf{I}, \mathbf{J}_{L}\right)=81.90 \%$ and $\Phi_{1}^{g}\left(\mathbf{I}, \mathbf{J}_{M}\right)=0.17 \%$.

\subsection{Average of Local Irregularity Indexes}

Despite its mathematical formulation, computing the global irregularity index is not an easy task for natural images. Precisely, this irregularity index requires solving a linear programming problem with $m n$ variables, where $m$ and $n$ are the number of distinct pixel values of the images $\mathbf{I}$ and $\mathbf{J}$, respectively. In practical situations, the dimension of the linear programming problem (8) is extremely large, making it impossible to be solved in real time. To circumvent this computational burden, we propose to compute the Wasserstein metric and the generalized sum of pixel-wise distances locally and aggregate the values into a single quantitative index.

Let $\left\{W_{1}, W_{2}, \ldots, W_{k}\right\}$, with $W_{\ell} \subseteq D$ for all $\ell=1, \ldots, k$, be a family of possibly overlapping local windows such that $D \subseteq \cup_{i=1}^{k} W_{i}$. Also, let $\mathcal{D}_{p}\left(\mathbf{I}, \mathbf{J} \mid W_{\ell}\right)$ and $\mathcal{W}_{p}\left(\mathbf{I}, \mathbf{J} \mid W_{\ell}\right)$ denote the generalized sum of pixel-wise distances and the Wasserstein metric computed restricting the images $\mathbf{I}$ and $\mathbf{J}$ to the local window $W_{\ell}$. The average of local irregularity indexes is defined by the following equation for all $\mathbf{I}, \mathbf{J} \in \mathcal{V}$ :

$$
\Phi_{p}^{a}(\mathbf{I}, \mathbf{J})=1-\left(\prod_{\ell=1}^{k} \frac{\mathcal{W}_{p}\left(\mathbf{I}, \mathbf{J} \mid W_{\ell}\right)}{\mathcal{D}_{p}\left(\mathbf{I}, \mathbf{J} \mid W_{\ell}\right)}\right)^{\frac{1}{p}}
$$

We would like to emphasize that, because the irregularity index is given by a ratio, the geometric mean is used to aggregate the quotient of $\mathcal{W}_{p}\left(\mathbf{I}, \mathbf{J} \mid W_{\ell}\right)$ by $\mathcal{D}_{p}\left(\mathbf{I}, \mathbf{J} \mid W_{\ell}\right)$.

In our computational implementation, inspired by the structural similarity index (SSIM) [24], we used local square windows of size $8 \times 8$ with strides of 4 pixels. Using $p=1$ and the Euclidean distance, the average of local irregularity indexes of the dilated images shown in Figure 1a) and 1c) are respectively $\Phi_{1}^{a}\left(\mathbf{I}, \mathbf{J}_{L}\right)=15.27 \%$ and $\Phi_{1}^{a}\left(\mathbf{I}, \mathbf{J}_{M}\right)=0.03 \%$. Note that $\Phi_{1}^{a}\left(\mathbf{I}, \mathbf{J}_{L}\right)$ is significantly less than $\Phi_{1}^{g}\left(\mathbf{I}, \mathbf{J}_{L}\right)=$ $81.90 \%$. However, the following experiment shows that the average of local irregularity indexes is highly correlated to the global irregularity index.

\subsection{The Global Irregularity Index and the Average of Local Irregularity Indexes}

Let us compare the global irregularity index and the average of local irregularity indexes using toy images similar to the one provided by Chevallier and Angulo [5] but with 
different probabilities. Precisely, we first construct an image of size $64 \times 32$ with two stripes of the same width but with the colors black $\boldsymbol{u}=(0,0,0)$ and blue $\boldsymbol{v}=(0,0,1)$ in the RGB color space. Then, a pure black pixel value is replaced by the black $\boldsymbol{w}=$ $(0.005,0,0)$ with probability $\pi \in[0,1]$. The resulting image $\mathbf{I}$ is dilated by a cross structuring element using both the lexicographical and the marginal ordering schemes. The simulation has been repeated 100 times for each probability $\pi \in[0,1]$. Figure 2 shows the mean of both the global irregularity index and the average of local irregularity indexes by the probability $\pi$. Again, we used the Euclidean distance to compute the cost to transform pixel values. For a better interpretation of this graph, dilated images obtained using the lexicographical ordering from images generated with probabilities $\pi=0.0,0.25,0.50,0.75$, and 1.0 are shown at the bottom of Figure 2 .

Note that both the global irregularity index and the average of the local indexes are very close to zero for the marginal ordering. In contrast, using the lexicographical RGB ordering, both irregularity indexes increase until close to $\pi=0.3$ and then decrease. Furthermore, the irregularity indexes agree with the visual irregularity provided in the sample images at the bottom of Figure 2. Finally, we would like to point out that the correlation between the global and average of local irregularity indexes is $98.77 \%$. Therefore, although they have different scales, we believe both the global irregularity index and the average of the local index can be used as an effective measure for the irregularity issue.

\subsection{Example with Natural Color Images}

Let us now provide some examples with natural color images. Precisely, we compute the average of local irregularity indexes $\Phi_{p}^{a}$, the generalized sum of pixel-wise distances $\mathcal{D}_{p}$, and the average of local Wasserstein metrics, denoted by $\mathcal{W}_{p}^{a}$, for several RGB images of size $256 \times 256$. Because of their computational burden, we refrained to compute the global irregularity index and the Wasserstein metric globally. In contrast to $\Phi_{p}^{a}$, however, the local Wasserstein metrics are aggregated using the arithmetic mean. Moreover, for better scaling the $\mathcal{D}_{p}$ and $\mathcal{W}_{p}^{a}$, these quantities have been divided by the number of pixels of the processed image. Like in the previous examples, we used the Euclidean distance as the metric of the value set. The quantitative measures have been computed using the erosion, the dilation, the opening, and the closing by a $7 \times 7$ square structuring element. Two approaches based on the total ordering have been considered: One based on the RGB lexicographical order and the other based on a supervised reduced ordering [19]. In a supervised reduced ordering, the pixel values are ranked using a supervised machine learning technique trained on a set of background and foreground pixels. We also included the marginal approach for comparison purposes. Recall that the marginal approach is not based on a total order. Thus, it can circumvent the irregularity issue.

Figure 3 summarizes the outcome of this computational experiment. To facilitate the exposition, the average of the local irregularity indexes, the generalized sum of pixelwise distances divided by the number of pixels of the image, and the average of local Wasserstein metric divided by the number of pixels of the local windows, are presented as a triple $\left(\Phi_{p}^{a}, \mathcal{D}_{p}, \mathcal{W}_{p}^{a}\right)$ below the output images in percentage.

The weakness of both the generalized sum of pixel-wise distances $\mathcal{D}_{p}$ and the Wasserstein metric $\mathcal{W}_{p}$ for measuring the irregularity is observed on the images shown 


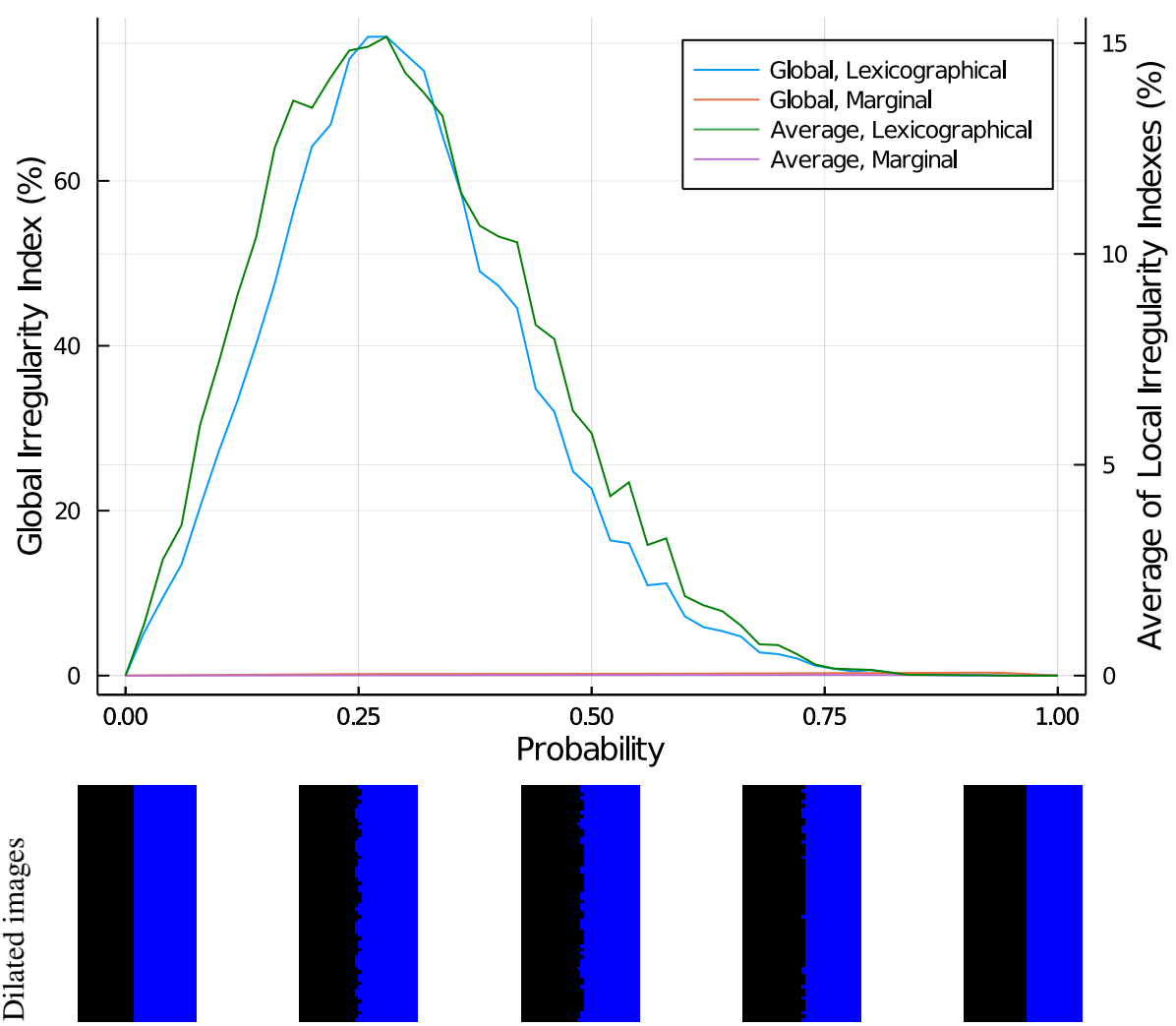

Fig. 2. Top: Irregularity indexes by the probability of replacing a pure black pixel value $\boldsymbol{u}=$ $(0,0,0)$ by the black $\boldsymbol{w}=(0.005,0,0)$ in an image similar to Figure $1 b)$. Bottom: Lexicographical dilation of images obtained using $\pi=0.0,0.25,0.5,0.75$, and 1.0 , respectively.

in Figure 3. As pointed out previously, $\mathcal{D}_{p}$ and $\mathcal{W}_{p}$ are not dimensionless measures; they are possibly measured using some photometric quantity such as lumen [17]. As a consequence, they may yield misleading values. For example, comparing the eroded boats and the dilated tulips depicted respectively in Figure $3 b$ ) and $3 f$ ), one may conjecture that the larger $\mathcal{D}_{p}$ or $\mathcal{W}_{p}$, the more irregular is the image. However, opening the Lena image or closing the balloons images using the lexicographical ordering, yielded the irregular images shown in Figure 3j) and $3 n$ ) whose quantities $\mathcal{D}_{1}$ and $\mathcal{W}_{1}^{a}$ are much smaller than the values obtained for the eroded boat images.

Let us now address the proposed irregularity index $\Phi_{1}^{a}$, which is a dimensionless measure. Note that the three eroded versions of the boat image shown in Figure 3b), $3 c)$, and 3d) are quite similar. Accordingly, the corresponding irregularity indexes have similar magnitudes. In contrast, the dilated version of the tulips image, depicted in 3f), $3 \mathrm{~g}$ ), and $3 \mathrm{~h}$ ), are quite different. In particular, the irregularities of the red pixels in the flowers on Figure 3f) obtained using the lexicographical ordering is noticeable. In agreement, the irregularity index $\Phi_{1}^{a}$ is much larger for the lexicographical ordering than 
a) Boat

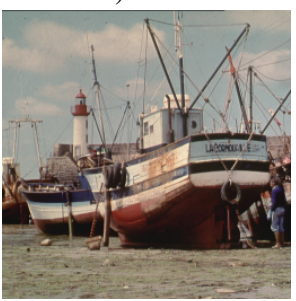

$\left(\Phi_{1}^{a}, \mathcal{D}_{1}, \mathcal{W}_{1}^{a}\right):$ b) Erosion - Lex.

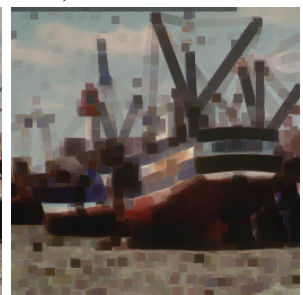

$(1.2 \%, 22.9 \%, 22.7 \%)$ c) Erosion - Superv.

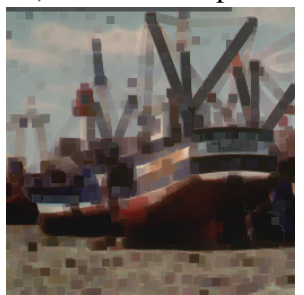

$(1.9 \%, 22.7 \%, 22.4 \%)$ d) Erosion - Marg.

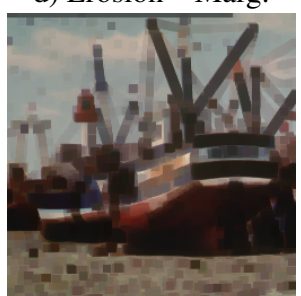

$(0.7 \%, 23.2 \%, 23.3 \%)$ e) Tulips

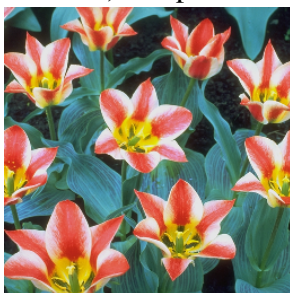

$\left(\Phi_{1}^{a}, \mathcal{D}_{1}, \mathcal{W}_{1}^{a}\right):$ f) Dilation - Lex.

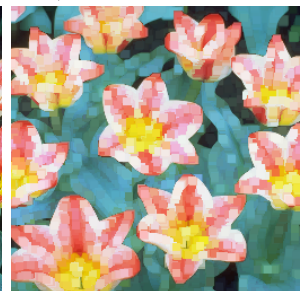

$(8.3 \%, 31.8 \%, 29.6 \%)$ g) Dilation - Superv.

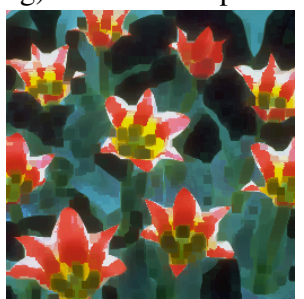

$(7.4 \%, 28.1 \% 26.3 \%)$ h) Dilation - Marg.

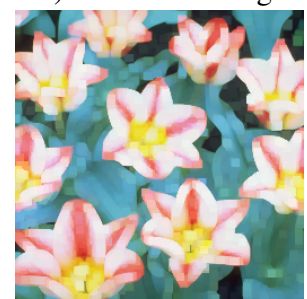

$(1.5 \%, 37.1 \%, 36.7 \%)$

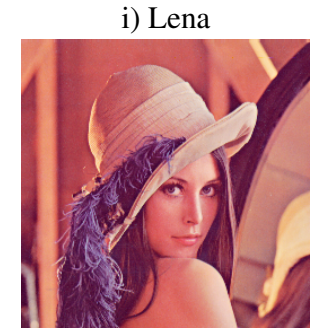

$\left(\Phi_{1}^{a}, \mathcal{D}_{1}, \mathcal{W}_{1}^{a}\right)$ :

m) Ballons

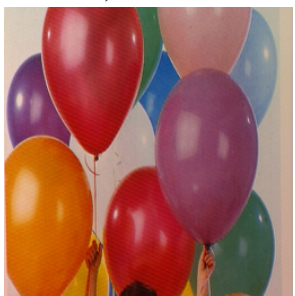

$\left(\Phi_{1}^{a}, \mathcal{D}_{1}, \mathcal{W}_{1}^{a}\right):$ j) Opening - Lex.

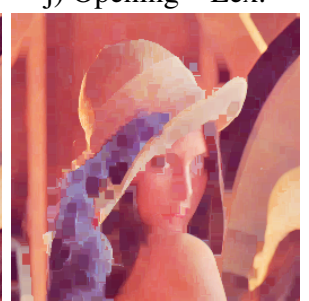

$(16.5 \%, 8.5 \%, 7.8 \%)$

n) Closing - Lex.

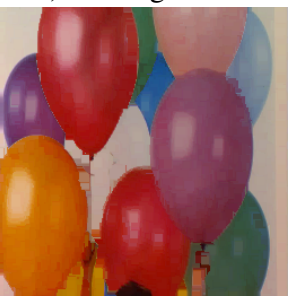

$(13.8 \%, 4.5 \%, 3.8 \%)$ k) Opening - Superv.

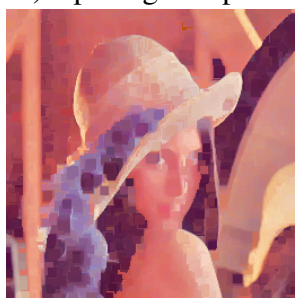

$(13.3 \%, 9.1 \%, 8.3 \%)$

p) Closing - Superv.

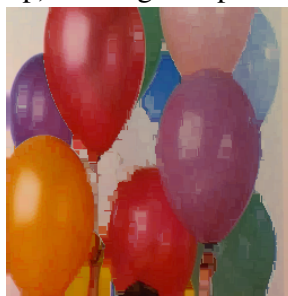

$(16.5 \%, 4.8 \%, 4.0 \%)$
1) Opening - Marg.

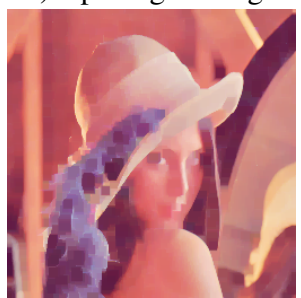

$(2.7 \%, 9.4 \%, 9.3 \%)$

o) Closing - Marg.

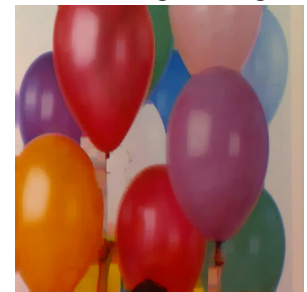

$(4.46 \%, 4.51 \%, 4.22 \%)$

Fig. 3. Illustrative examples of the average of local irregularity indexes $\Phi_{1}^{a}$ computed for several color images using different morphological operators. For comparison purposes, we also included the sum of pixel-wise distances $\mathcal{D}_{1}$ and the average of local Wasserstein metric $\mathcal{W}_{1}^{a}$. 
for the marginal ordering. Similar remarks hold by comparing the irregularity indexes obtained for the images $3 \mathrm{j}$ ), 3k), and 3l) or 3m), 3n), and 3o). In fact, except for the boat image, the irregularity index obtained for the lexicographical and supervised approaches are significantly larger than the irregularity index yielded by the marginal approach.

We invite the reader to carefully compare the images and the corresponding quantities shown in Figure 3. We hope this example will help the reader to be convinced of the superiority of the proposed irregularity index, in particular, over both the generalized sum of pixel-wise distances and the Wasserstein metric.

\section{Concluding Remarks}

In this paper, we proposed two quantitative measures for the irregularity issue. Namely, the global irregularity index and the average of the local irregularity indexes, denoted respectively by $\Phi_{p}^{g}$ and $\Phi_{p}^{a}$. Although Chevallier and Angulo provided a rigorous formulation of the irregularity issue [5], as far as we know, there is no effective quantitative measure for this problem. For example, the generalized sum of pixel-wise distance $\mathcal{D}_{p}$, which is closely related to the norm of (internal or external) gradient or top-hat operations, is not a dimensionless measure [16,15]. Similarly, the Wasserstein metric $\mathcal{W}_{p}$, which is eventually known as the earth mover's distance, is also not a dimensionless measure [11]. Hence, they may not be appropriately scaled. Accordingly, as can be observed in Figure 3, both $\mathcal{D}_{p}$ and $\mathcal{W}_{p}$ are not appropriate for measuring the irregularity issue. In contrast, the dimensionless global irregularity index given by the relative gap between $\mathcal{D}_{p}$ and $\mathcal{W}_{p}$ yielded good quantitative values for the irregularity for images with few pixel values. In practical situations, however, the number of distinct pixel values makes it impossible to compute the global irregularity index. To circumvent this computational drawback, we proposed computing the geometric mean of the irregularity index on several small windows. Visual interpretations of the irregularity indexes are provided using both synthetic and natural images.

Finally, we would like to point out that the irregularity index can be used in the future to evaluate the performance of morphological operators. The irregularity index can also be used for the design of efficient morphological operators. For example, it can be used as the objective function for the design of vector-valued morphological operators based on uncertain reduced orderings [13].

\section{References}

1. Angulo, J.: Morphological colour operators in totally ordered lattices based on distances: Application to image filtering, enhancement and analysis. Computer Vision and Image Understanding 107(1-2), 56-73 (2007)

2. Aptoula, E., Lefèvre, S.: A Comparative Study on Multivariate Mathematical Morphology. Pattern Recognition 40(11), 2914-2929 (2007)

3. Birkhoff, G.: Lattice Theory. American Mathematical Society, Providence, 3 edn. (1993)

4. Burgeth, B., Kleefeld, A.: An approach to color-morphology based on Einstein addition and Loewner order. Pattern Recognition Letters 47(0), 29-39 (2014) 
5. Chevallier, E., Angulo, J.: The Irregularity Issue of Total Orders on Metric Spaces and Its Consequences for Mathematical Morphology. Journal of Mathematical Imaging and Vision 54(3), 344-357 (3 2016). https://doi.org/10.1007/s10851-015-0607-7

6. Dougherty, E.R., Lotufo, R.A.: Hands-on Morphological Image Processing. SPIE PRESS (2003)

7. van de Gronde, J., Roerdink, J.: Group-Invariant Colour Morphology Based on Frames. IEEE Transactions on Image Processing 23(3), 1276-1288 (2014)

8. Heijmans, H.J.A.M.: Mathematical Morphology: A Modern Approach in Image Processing Based on Algebra and Geometry. SIAM Review 37(1), 1-36 (1995)

9. Lézoray, O.: Complete lattice learning for multivariate mathematical morphology. Journal of Visual Communication and Image Representation 35, 220-235 (2016). https://doi.org/10.1016/j.jvcir.2015.12.017

10. Ronse, C.: Why Mathematical Morphology Needs Complete Lattices. Signal Processing 21(2), 129-154 (1990)

11. Rubner, Y., Tomasi, C., Guibas, L.J.: Earth mover's distance as a metric for image retrieval. International Journal of Computer Vision 40(2), 99-121 (11 2000). https://doi.org/10.1023/A:1026543900054, https://link.springer.com/article/10.1023/A:1026543900054

12. Sangalli, M., Valle, M.E.: Color Mathematical Morphology Using a Fuzzy Color-Based Supervised Ordering. In: Barreto, G.A., Coelho, R. (eds.) Fuzzy Information Processing. pp. 278-289. Springer International Publishing (2018)

13. Sangalli, M., Valle, M.E.: Approaches to Multivalued Mathematical Morphology Based on Uncertain Reduced Orderings. In: Burgeth, B., Kleefeld, A., Naegel, B., Passat, N., Perret, B. (eds.) Mathematical Morphology and Its Applications to Signal and Image Processing. pp. 228-240. Springer International Publishing, Cham (2019). https://doi.org/10.1007/9783-030-20867-7_18

14. Serra, J.: The "False Colour" Problem. In: Wilkinson, M.H.F., Roerdink, J.B.T.M. (eds.) Mathematical Morphology and Its Application to Signal and Image Processing, Lecture Notes in Computer Science, vol. 5720, pp. 13-23. Springer Berlin Heidelberg (2009)

15. Soille, P.: Morphological Image Analysis. Springer Verlag, Berlin (1999)

16. Soille, P., Vogt, J., Colombo, R.: Carving and adpative drainage enforcement of grid digital elevation models. Water Resources Research 39(12), 1366 (2003)

17. Trussell, H.J., Vrhel, M.J.: Photometry and colorimetry. In: Fundamentals of Digital Imaging, pp. 191-244. Cambridge University Press, Cambridge (2008). https://doi.org/10.1017/CBO9780511754555.009

18. Valle, M.E., Valente, R.A.: Mathematical Morphology on the Spherical CIELab Quantale with an Application in Color Image Boundary Detection. Journal of Mathematical Imaging and Vision 57(2), 183-201 (2 2017). https://doi.org/10.1007/s10851-016-0674-4, https://link.springer.com/article/10.1007/s10851-016-0674-4

19. Velasco-Forero, S., Angulo, J.: Supervised Ordering in Rp: Application to Morphological Processing of Hyperspectral Images. IEEE Transactions on Image Processing 20(11), 33013308 (11 2011). https://doi.org/10.1109/TIP.2011.2144611

20. Velasco-Forero, S., Angulo, J.: Random Projection Depth for Multivariate Mathematical Morphology. IEEE Journal of Selected Topics in Signal Processing 6(7), 753-763 (11 2012). https://doi.org/10.1109/JSTSP.2012.2211336

21. Velasco-Forero, S., Angulo, J.: Mathematical Morphology for Vector Images Using Statistical Depth. In: Soille, P., Pesaresi, M., Ouzounis, G.K. (eds.) Mathematical Morphology and Its Applications to Image and Signal Processing. pp. 355-366. Springer Berlin Heidelberg, Berlin, Heidelberg (2011) 
22. Velasco-Forero, S., Angulo, J.: Vector Ordering and Multispectral Morphological Image Processing. In: Celebi, M.E., Smolka, B. (eds.) Advances in Low-Level Color Image Processing. pp. 223-239. Springer Netherlands, Dordrecht (2014). https://doi.org/10.1007/978-94-0077584-8_7

23. Villani, C.: Topics in Optimal Transportation. American Mathematical Society, Providence, Rhode Island (2003)

24. Wang, Z., Bovik, A.C., Sheikh, H.R., Simoncelli, E.P.: Image quality assessment: From error visibility to structural similarity. IEEE Transactions on Image Processing 13(4), 600-612 (2004) 